\title{
INFORMAÇÃO/EDUCAÇÃO EM DST/AIDS/SEXUALIDADE HUMANA - UM RELATO DE EXPERIÊNCIA DE 14 ANOS
}

\section{INFORMATION/EDUCATION IN STD/AIDS/HUMAN SEXUALITY - A CASE REPORT AFTER 14 YEARS OF EXPERIENCE}

\author{
Elucir Gir* \\ Tokico Murakawa Moriya* \\ Maria Helena Pessini de Oliveira* \\ Nilza Teresa Rotter Pelá**
}

GIR, E. et al. Informação/educaçăo em DST/Aids/sexualidade humana - um relato de experiência de 14 anos. Rev.Esc.Enf.USP, v.32, n.4, p. 291-96, dez.1998.

\begin{abstract}
RESUMO
As autoras relatam suas experiências de 14 anos sobre informação/educação em Doenças Sexualmente Transmissíveis/ Aids e Sexualidade Humana. Descrevem as ações implementadas, destacando os resultados positivos, bem como os negativos. Quanto aos resultados positivos destacam evidentemente a divulgação de informações sobre medidas preventivas acerca da infecção pelo HIV/AIDS, DST e aspectos da sexualidade humana a diversos segmentos da comunidade leiga e cientifica, bem como, o repasse do aprendizado adquirido nessas vivencias para o ensino de graduação, pós-graduação e desenvolvimento da pesquisa. Quanto aos negativos, perceberam a dificuldade em avaliar as ações de informações, ou seja palestras, por constituírem-se uma estratégia de comunicaşão praticamente unilateral. Nessa trajetória apontam alguns fatos que historicamente marcaram a implementação de ações o, ficiais.
\end{abstract}

UNITERMOS: Informação. Educação. Sexualidade humana. Doenças sexualmente transmissíveis.

\section{ABSTRACT}

The authors present their 14-year experience about Information/education on sexually transmitted diseases/Aids and human sexuality. They describe the actions inplemented, emphasizing the positive and negative points. Concerning the positive results they mention the divulgation of information about preventive measures against HIV infection/ Aids, Sexually Transmitted Diseases and human sexuality to several people from scientific and non scientific community. Another positive point is the transmission of knowledge gotten in such experiences at undergraduation and graduation teaching, as well as the development of research. Concerning the negative aspects, the difficult to evaluate the information actions, specially lectures was perceived, mainly because it is a communication tool basically unilateral. The authors mention some facts that historically influenced the implementation of the official actions.

UNITERMS: Information. Education. Human sexuality. Sexually transmitted diseases.

\section{INTRODUÇÃO}

Preocupados com o aumento das Doenças Sexualmente Transmissíveis (DST) a partir da década de 60, reuniu-se na Escola de Enfermagem de Ribeirão Preto - USP, um grupo de cinco enfermeiras docentes que tinham como proposta de trabalho divulgar informações sobre a prevenção e o controle destas doenças.
Esta proposta emanada em 1982, proporcionou em primeiro momento a sensibilização e a atualização do pessoal da área de saúde, do município de Ribeirão Preto - SP, sobre a temática.

O processo estava em desenvolvimento quando uma nova entidade clínica de transmissão freqüentemente sexual, a Acquired Immune

\footnotetext{
* Enfermeira,. Docente da Escola de Enfermagem de Ribeirão Preto-USP

** Enfermeira, Docente da Escola de Enfermagem de Ribeirão Preto-USP (aposentada)
} 
Deficiency Syndrome (Aids), surpreendeu a humanidade pelo seu perfil de alta morbimortalidade, a inexistência de terapêutica específica, pelo potencial devastador de disseminação e agravos psicossociais.

Nesse momento, o mencionado grupo entendeu a necessidade de trabalhar divulgando informações sobre as formas de transmissão e medidas de prevenção, oficializando-se o grupo de trabalho com o nome de Núcleo de Aids e Doenças Sexualmente Transmissíveis (NAIDST), em 1985. A gênese e o desenvolvimento das ações de informação/ educação tinham, então como objetivos o desenvolvimento de ensino, pesquisa e assistência em DST/Aids. Ressalta-se que a promoção das atividades realizadas com um enfoque de prevenção através da passagem de informações constituíram o objetivo inicial do grupo. Em conseqüência do processo informativo e educativo os profissionais começaram também a desenvolver investigações científicas sobre a temática em questão, o que resultou em 1990 ser o NAIDST cadastrado junto aos Grupos de Pesquisa do Brasil no Conselho Nacional de Pesquisa (CNPq).

Concomitantemente ocorreu um movimento de reorganização das práticas de Prevenção e ao Controle do HIV/Aids através da elaboração e divulgação de normas por órgãos governamentais. como Ministério da Saúde e Secretarias Municipais de Saúde.

Em Ribeirão Preto, oficialmente, o programa de Prevenção e Controle da AIDS teve seu início em fevereiro de 1987. Envolveram-se neste programa várias instituições, ou seja a Secretaria do Estado da Saúde, a Escola de Enfermagem de Ribeirão Preto -USP, a Faculdade de Medicina de Ribeirão Preto USP, o Hospital das Clínicas da Faculdade de Medicina de Ribeirão Preto - USP, a Divisão Regional de Ensino, a Secretaria Municipal da Educação, constituindo-se um grupo interinstitucional de prevenção e controle da Aids.

A experiência vivenciada por nós, enfermeiras, docentes da Escola de Enfermagem de Ribeirão Preto - USP, no serviço de informação ao público, que consistiu em ministração de aulas, palestras, cursos, conferências, desde sua implantação foi bastante enriquecedora. Assim, com o objetivo de relatar a experiência de 14 anos (1982-1995), quatro dos cinco elementos deste grupo se propuseram escrever a experiência vivenciada, com a finalidade de divulgar a experiência e oferecer subsídios para grupos que atuam em DST/Aids.

\section{METODOLOGIA}

Os dados foram levantados retrospectivamente a partir de registros contidos em um livro de atas do NAIDST e memoriais de docentes, referentes a palestras, aulas, conferências, cursos ministrados mediante convites da comunidade.

$O$ procedimento se deu através do levantamento de todas as atividades de informação/ educação sobre AIDS e DST desenvolvidas no período de 1982 a 1994, levando-se em conta:

- temática explorada,

- modalidade da atividade,

- tipo de instituição envolvida e sua localização e

- número de pessoas atingidas

\section{RESULTADOS E DISCUSSÃO}

Atendendo a solicitações de instituições, empresas e grupos da comunidade, os membros do NAIDST realizaram, no período de 1982 a 1995, 426 ações educativas (aulas, palestras, conferências e cursos).

Observa-se na Figura 1, que em 1987, um total de $70(16,43 \%)$ ações educativas e em 1988, 53 $(12,44 \%)$ ações foram realizadas, caracterizando estes dois anos como os de maior atuação do período relatado. 
Figura 1. Distribuição das ações informativas desenvolvidas pelos membros do NAIDST (Ribeirão Preto, 1982 a 1995).

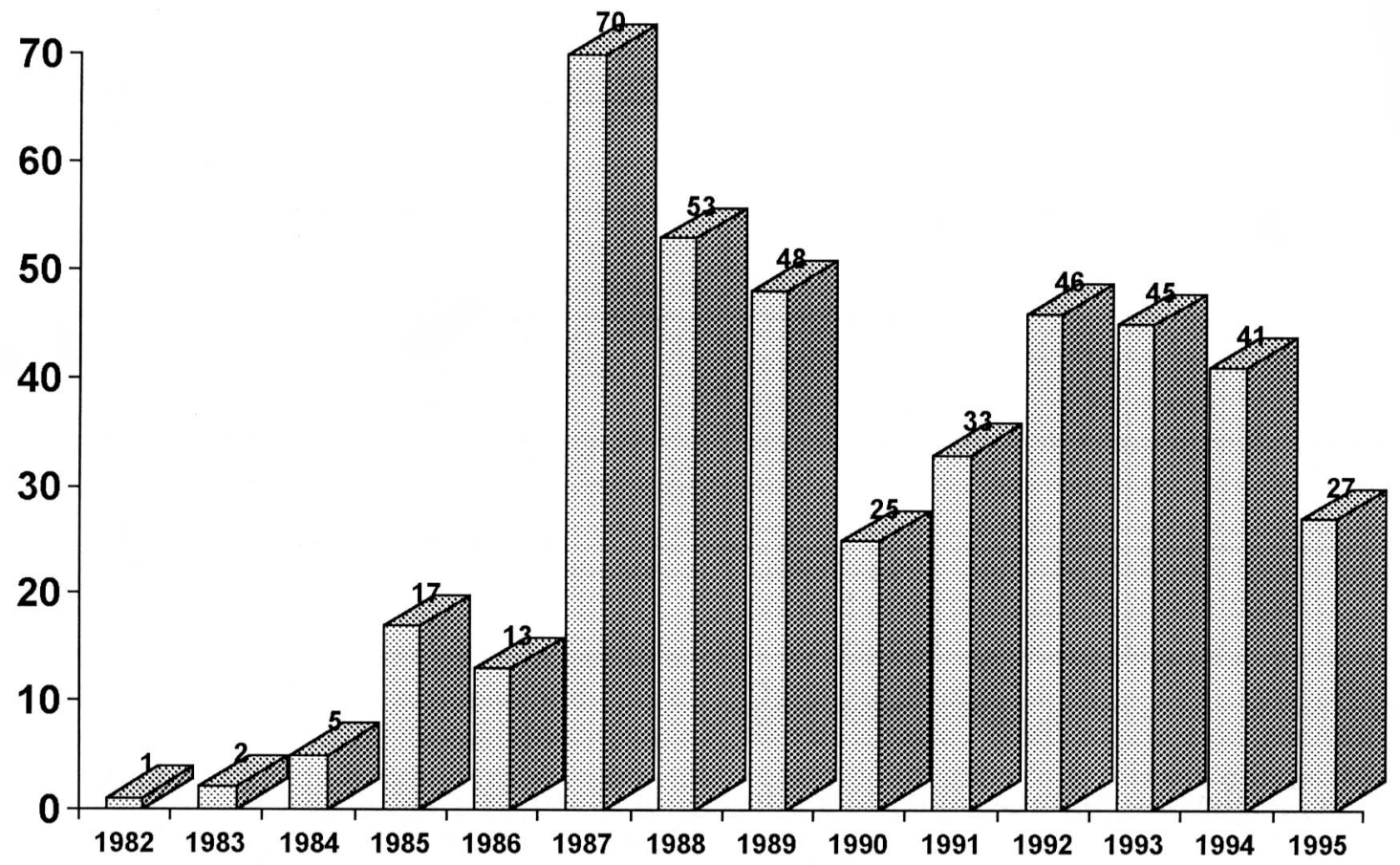

Nesse biênio as ações constituíram-se basicamente em palestras, sendo significativo o número de pessoas que foram expostas ao processo de informação. Esse dado é de certa forma obvio, se for levado em conta o respectivo aumento do número de palestras neste período, como consta na Figura 1.

Os destaques dirigidos para os anos de $1987 \mathrm{e}$ 1988 , como os mais profícuos quantitativamente, podem ser atribuídos, dentre outros fatores, ao reconhecimento da problemática da Aids pelos diversos segmentos da sociedade e portanto a necessidade de informações adequadas à população e à publicação da portaria Interministerial de $n^{\circ}$. 3195 de 10 de agosto de 1988, que instituiu em âmbito nacional a Campanha Interna de Prevenção da Aids nas empresas (AIDS, 1988).

Supõe-se que buscando atender à exigência estabelecida nessa portaria, as empresas passaram a solicitar ao grupo a realização de palestras e outras atividades educativas, oferecendo facilidades como transporte ao professor que as executasse e liberação dos funcionários para participarem.

Essa situação era decorrente, sobretudo, das exigências e facilidades que as empresas expunham seus funcionários em conseqüência à Portaria supra citada. A demanda crescente neste período foi intensamente atendida pelo grupo do NAIDST, que tinha a palestra como sua atividade mais relevante.

Este aspecto pôde ser sentido pelo grupo, pela própria atitude dos solicitantes, na realização das ações educativas. Nas instituições de ensino o empenho era feito para que, alunos e professores, participassem das palestras. Por outro lado, muitas empresas, solicitavam palestras, porém encaminhavam um número restrito de funcionários para assistirem as mesmas, pois não tinham interesse em capacitar multiplicadores de ações . Houve situações onde as empresas solicitavam inclusive um atestado de que havia sido realizada palestra sobre Aids. Este fato é confirmado, em parte pelos dados contidos no Quadro 1. Observa-se que das 426 (100\%) atividades realizadas, houve destaque para as desenvolvidas em empresas (161$37,79 \%$ ), seguida das realizadas em instituições educacionais $(117 \cdot 27,46 \%)$. É sabido que a região de Ribeirão Preto, é um polo econômico onde se concentram várias empresas usineiras de açúcar e álcool, bem como um importante centro universitário. 
Quadro I - Distribuição das atividades informativas/educativas realizadas pelo NAIDST (Ribeirão Preto, 1982 - 1995), segundo o ano e local atendido

\begin{tabular}{|c|c|c|c|c|c|c|c|c|c|c|c|c|c|c|c|}
\hline $\begin{array}{c}\text { Tipo de } \\
\text { local }\end{array}$ & 1982 & 1983 & 1984 & 1985 & 1986 & 1987 & 1988 & 1989 & 1990 & 1991 & 1992 & 1993 & 1994 & 1995 & Total \\
\hline Empresa & - & 1 & 2 & 4 & 6 & 26 & 31 & 28 & 12 & 8 & 10 & 12 & 15 & 6 & $161(37,79)$ \\
\hline $\begin{array}{l}\text { Instituição } \\
\text { de Ensino }\end{array}$ & - & - & 1 & 7 & 4 & 30 & 8 & 7 & 4 & 13 & 9 & 18 & 11 & 5 & $117(27,46)$ \\
\hline $\begin{array}{l}\text { Instituição } \\
\text { de Saúde }\end{array}$ & 1 & 1 & . & 3 & 1 & 8 & 8 & 5 & 5 & 9 & 17 & 10 & 7 & 15 & $90(21,13)$ \\
\hline $\begin{array}{l}\text { Grupamen } \\
\text { Específico } \\
*\end{array}$ & - & - & 2 & 3 & 2 & 6 & 6 & 8 & 4 & 3 & 10 & 5 & 8 & 1 & $58(13,61)$ \\
\hline
\end{tabular}

* Destacamento de polícia, tiro de guerra, FEBEn , cadeia pública.

Embora seja relevante numericamente o fato de 19.000 pessoas terem sido expostas às informações, através de palestras, o grupo iniciou discussões acerca do valor e adequação desta estratégia para se obter mudanças de comportamentos, pois para que os indivíduos substituam comportamentos inseguros por seguros é preciso haver sensibilização dos mesmos quanto à problemática e decisão própria e para tanto os elementos afetivos e cognitivos precisam ser trabalhados, aspectos não priorizados neste tipo de atividade. Além do mais, a palestra configurava-se como um momento único de contato entre o professor e a clientela, não viabilizando discussão e avaliação da ação.

Observa-se na Figura 1 e Quadro 1 declínio de freqüência de atividades em 1990 e 1991, devido a não aceitação de todos os convites recebidos. Tal fato teve como motivos principais a inquietação e percepção do grupo quanto à adequação da estratégia até então utilizada e a percepção de que as empresas deveriam estar instrumentalizando-se com recursos humanos capacitados para atender as suas necessidades internas de prevenção e controle de doenças. Ressalta-se que esse grupo de enfermeiras, autoras do presente trabalho, também foram responsáveis pelo estímulo e sensibilização das empresas quanto a este aspecto. Neste momento as atividades tiveram enfoque para a capacitação de multiplicadores de ações educativas, em substituição à ministração de palestras.

Apesar de reconhecer a limitação da palestra enquanto estratégia de ensino, ao se limitar à simples transmissão de informações, ainda acreditase que ela viabiliza aproximação sucessiva com a temática, facilitando futuras abordagens mais profundas com vistas à mudanças de comportamentos. Além disso, esta estratégia se fez necessária no momento inicial da epidemia, em que a divulgação de informações corretas sobre a doença foi fundamental frente a explosiva carga de tabus, mitos, preconceitos e crenças, que acompanharam o surgimento da Aids.

Conforme refere POMPIDOU (1988: 31) "estar informado não significa necessariamente conhecer, estar ciente, não significa necessariamente tomar medidas, decidir não necessariamente quer dizer fazer"; assim, a informação por si só não basta para provocar mudanças de comportamentos.

Portanto, embora o conhecimento seja um dos aspectos fundamentais para provocar mudanças de atitudes ou comportamentos, por outro lado, sabe-se que muitas pessoas resistem ou ignoram o conhecimento adquirido sobre transmissão e prevenção da infecção pelo HIV, por ocasião dos eventos das relações sexuais e uso endovenoso de drogas ilícitas, subestimando a SUA probabilidade em infectar-se e acreditando que a Aids está distante de si e que não vai acontecer consigo. Certamente que questões referentes ao comportamento sexual são complexas porque muitas vezes o indivíduo compreende a situação, porém não consegue introjetar ou colocar em prática o que a ciência comprova, com vistas à promoção à saúde (GIR et al., 1994). 
A mudança de atitudes, sobretudo é gerada a partir da introjeção do senso de responsabilìdade despertado e assumido pelo indivíduo e não por imposição ou obrigação (POMPIDOU, 1988).

Conforme refere VITIELLO (1994) o Informar é um dos componentes do Educar, sendo que o Educar não só engloba o Informar, Aconselhar, Orientar, bem como provoca mudanças interiores no indivíduo. Informação é "o mero fornecimento de fatos e dados. Leva a um crescimento individual por aposição, de fora para dentro. Mesmo considerando que possa fazer parte do processo de educação, não o substitui" (p. 222). Aconselhamento consiste em "auxiliar alguém"a escolher uma das opções disponíveis" (p. 211). Orientação é um "processo pelo qual se auxilia uma pessoa a analisar quais as opções de escolha de que dispõe" (p.225). Educação consiste em "fornecer a alguém subsídios e modelos para o crescimento pessoal e para a assunção de ideais e de comportamentos próprios. É um processo complexo, que requer tempo e influência de pessoas significativas" (p. 216).

Frente a estas considerações passam então os componentes do NAIDST a canalizarem grande parte de seu tempo e esforço no sentido de contribuir com a formação dos profissionais da saúde para atenderem a demanda de empresas e outras instituições e grupos quanto ao processo informativo, destacando-se nesse período (1992-1995) a maior ocorrência de realização de cursos e não de palestras desvinculadas de continuidade e processos avaliativos.
Observando-se na Figura 2 a freqüência de atividades educativas segundo a modalidade e a temática abordada, pode-se verificar maior número de atividades em cursos do que em palestras isoladas. Essa dinâmica foi decorrente do amadurecimento do grupo a partir das experiências vivenciadas, que reconhecem a necessidade de trabalhar com uma mesma população em várias etapas estimulando a discussão e o aprofundamento do nível de informações. Este quadro também evidencia a linha de pensamento do grupo, que as ações de seus membros não devem ser restritas unicamente à transmissão de conhecimentos, mas sobretudo à formação de agentes multiplicadores. Um outro aspecto que deve ser lembrado é que o "Informar" limita a troca de experiência, a discussão sobre dúvidas e esclarecimentos. A transmissão de mensagens é unidirecional, enquanto o "Educar" envolve a troca de mensagens, e este processo bidirecional é altamente rico, principalmente para se desfazer mensagens recebidas erroneamente e com ruídos.

Chama-se atenção ainda, para as temáticas Doenças Sexualmente Transmissíveis (DST) e Sexualidade. DST é por nós reconhecida como temática de grande interesse e importância desde o início da década de 80 . Sexualidade começou a fazer parte de nossas atividades em 1989, entretanto o começo da nossa capacitação específica em sexualidade humana foi em 1987.

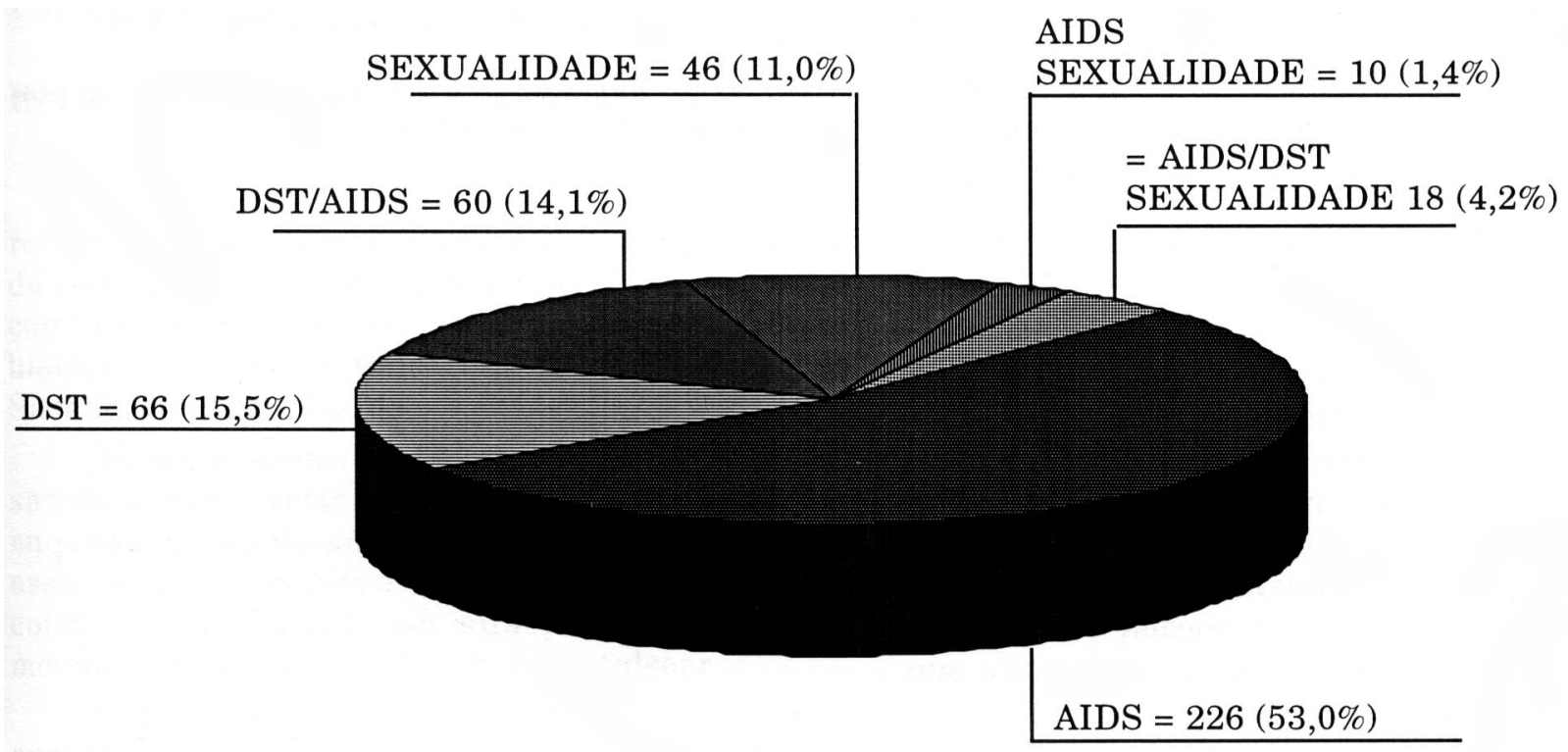

Figura 2 - Distribuição das temáticas desenvolvidas pelos membros do NAIDST, nas atividades informativas/educativas. Ribeirão Preto - SP, 1982 - 1995. 
O Ministério da Saúde, contudo, reconheceu que Aids e as outras DST não deveriam ser trabalhadas isoladamente, somente no final da década de 80. Começou a recomendar que quaisquer ações sobre Aids deveriam ser integradas as de DST, e vice-versa. A partir daí, a dissociação dessas temáticas não se fazem pertinentes, principalmente pela comprovação de que as DST erosivas constituem-se fator de risco expressivo para a aquisição do HIV.

A abrangência das ações desenvolvidas pelo núcleo como foco irradiador foi de aproximadamente $200 \mathrm{Km}$ de diâmetro atingindo 15 municípios tanto da região administrativa da região de Ribeirão Preto, como fora dela.

Esse aspecto faz reportar à necessidade de que pessoas que compõem grupos dessa natureza tenham uma filosofia de compromisso geradora de disponibilidade interna para realização dessas atividades, que demandam dispêndio físico, mental, econômico.

\section{CONSIDERAÇÕES FINAIS}

Esse olhar retrospectivo permite concluir que esses 14 anos de experiência em atividades informativas/educativas foram produtivos enquanto prestação de serviços à comunidade, principalmente no momento crítico dos primeiros anos de divulgação sobre a infecção pelo HIV. O período inicial foi acompanhado de pânico, incertezas, reacendendo preconceitos, atitudes discriminatórias, dúvidas, mas também viabilizando o crescimento individual e coletivo, tanto que no presente momento o grupo apresenta-se mais consolidado, preservando-se as características típicas daquelas de sua origem, viabilizando novas estratégias de trabalho. Essa vivência proporcionou também aos membros do NAIDST contatos sucessivos com problemas que geraram investigações científicas como conseqüência destas atividades de campo, sendo as atividades de pesquisa e de ensino enriquecidas. Hoje, além do conteúdo DST/Aids fazerem parte do currículo de graduação, foram criadas disciplinas em nivel de pósgraduação - mestrado e doutorado, cursos de treinamentos e atualização. Além das diversas publicações de trabalhos científicos já divulgados, sobre o tema, numerosas dissertações, teses, trabalhos de iniciação científica têm sido realizados sob as temáticas DST/Aids sexualidade humana, sob orientação dos membros do NAIDS'T, fruto das vivências e estudos.

\section{REFERÊNCIAS BIBLIOGRÁFICAS}

AIDS e local de trabalho. Fundacentro: Atualidades em prevenção de acidentes, v.19, n. 226, p. 7-10, 1988.

GIR, E. et al. Práticas sexuais e a infecção pelo vírus da imunodeficiência humana. AB, Goiânia, 1994

POMPIDOU, A. National AIDS information programme in France. In: WORLD HEALTH ORGANIZATION. AIDS: prevention and control. Geneva, Pergamon Press, 1988. p. 28-31.

VITIELLO, N. Reprodução e sexualidade: um manual para educadores. São Paulo, CEICH, 1994. 